\title{
CLIMAT ESTIVAL ET DEPERISSEMENT DU BOULEAU A PAPIER ${ }^{1}$
}

\section{PAR MARCEL LORTIE, RENE POMERLEAU ET GERARD MICHAUD 2}

Gérard Michaud, diplomé de l'Ecole des Gardes forestières de Duchesnay en 1946, a travaillé au bureau de pathologie forestière, Ministère des Terres des Terres et Forêts de la Province de Québec jusqu'en 1952 et depuis lors il occupe un poste de technicien au Laboratoire de Recherches forestières de Québec.

\section{RESUME}

Au cours d'une étude sur les effets du microclimat dans un peuplement de bouleau à papier atteint de dépérissement, des périodes de température élevée et de sécheresse furent enregistrés en 1955 et en 1959. En aucun temps, la température du sol n'a atteint un point dangereux, mais l'humidité du sol s'est abaissée à des niveaux critiques pendant de courts laps de temps. Cependant, la desiccation passagère $d u$ sol n'a pas exercé une influence défavorable visible sur le comportement des 550 arbres sous observation pendant six années.

\section{ABSTRACT}

During a six-year study of the microclimate in relation to the dieback of white birch, periods of high temperature and drought were recorded in 1955 and in 1959. At no time did the soil temperature reach a dangerous point but soil moisture fell to a critical level for short periods. However, no noxious effect that could be traced to temporary soil dryness was noted on the 550 trees under observation.

\section{INTRODUCTION}

En dépit des nombreux travaux effectués depuis au-delà de vingt ans sur le dépérissement généralisé et aigu du bouleau jaune (Betula alleghaniensis Britt.) et du bouleau à papier ( $B$. papyrifera Marsh.) dans l'est du Canada et le nord-est des Etats-Unis, beaucoup reste encore à faire pour élucider ce problème complexe. Il n'y a pas lieu de refaire ici une revue bibliographique de ce sujet déjà présentée par Redmond (1957) et Clark et Barter (1958). Toutefois, il importe de rappeler certains faits qui ont motivé nos recherches sur un aspect particulier de cette affection.

Jusqu'à maintenant, nul n'a pu démontrer péremptoirement qu'un insecte ou un parasite avait engendré ce dépérissement intense des bouleaux. Aussi, plusieurs chercheurs abordèrent-ils ce problème sous l'angle des rapports de la maladie avec le milieu ambiant. Dès 1944, Pomerleau émit l'opinion que cette mort-en-cime, apparue soudainement sur un vaste territoire, découlait de l'allure particulière du climat. Cette idée fut reprise par Hawboldt (1952) qui, par une étude climatologique, a pu montrer qu'un relèvement notable de la température moyenne d'été de 2 à $3^{\circ} \mathrm{F}\left(1^{\circ} \mathrm{C}\right)$ et un abaissement sensible et concomitant de la pluviosité ont précédé les premières manifestations de cette maladie. Redmond (1955) s'est engagé encore davantage dans cette voie et a

\footnotetext{
${ }^{1}$ Contribution No. 840, Direction de l'Entomologie et de la Pathologie forestières. Ministère des Forêts, Ottawa, Canada.

2 Laboratoire de Recherches forestières, C.P. 35, Sillery, Québec. Notices biographiques de deux premiers auteurs déja parues dans les numéros de décembre 1961 et de juin 1962.
} 
déduit que le réchauffement du climat devait se traduire par une augmentation correspondante de la température du sol. Pour étayer sa thèse, il a relevé artificiellement la température du sol d'environ $5^{\circ} \mathrm{F}\left(2^{\circ} \mathrm{C}\right)$ dans un peuplement de bouleau jaune et a noté que la proportion normale de 6 pour cent de radicelles mortes chez les jeunes arbres était passée à 60 pour cent à la fin de l'expérience. Toutefois, aucune trace de ce traitement n'a subsisté chez ces mêmes sujets les années suivantes (Redmond 1958). Ces résultats, qui n'apportèrent aucune solution même partielle au problème, ont cependant retenu l'attention et souligné l'intérêt d'en aborder l'étude sous cet angle. Dès lors, il importait de mieux connaître l'influence que pouvaient exercer la température et l'humidité du sol sur la santé des arbres. C'est précisément avec cette intention que nous avons entrepris en 1955, et poursuivi jusqu'en 1960 , une étude sur le comportement des bouleaux à papier en rapport avec le climat estival dans une station donnée dans la province de Québec.

STation d'OBservation

Pour effectuer ces travaux, nous avons recherché une station, plutôt uniforme en apparence, où un secteur de forêt relativement indemne avoisinait une partie plus fortement atteinte du dépérissement. A l'endroit choisi, à Saint-Tite-des-Caps, C. de Charlevoix, P. Qué., se trouvait un peuplement inéquienne mélangé à forte proportion de bouleau à papier d'environ 80 ans, probablement venu après un incendie, nullement altéré depuis et caractérisé par un tapis végétal à Cornus-Maianthemum (Linteau 1959). Le sol, constitué d'une couche d'humus de 2 à 3 pouces d'épaisseur, d'un horizon $A_{2}$ de 3 à 4 pouces et d'un horizon B composé de gravier et de limon sableux, brun rouillé, était bien drainé et avait un régime d'humidité d'une valeur de 2, selon la classification de Hills (1945).

\section{Methode D'Observation}

Dans cette station, nous avons délimité deux aires d'étude d'une demi-acre de superficie, l'une dans la partie la moins affectée (Aire A) et l'autre dans la partie fortement atteinte (Aire B). Dans chacune des parcelles, les bouleaux furent dénombrés, mesurés, et classifiés quant au dépérissement de la cime, selon la méthode de Hawboldt et Skolko (1948) (Tableau 1). Puis, chaque année, jusqu'en 1960 inclusivement, ces arbres furent classifiés selon le même barème. Pour faciliter la compilation et obtenir des moyennes, nous avons,

TABLEAU 1

DESCRIPTION SOMMAIRE ET EQUIVALENCE NUMERIQUE DES CLASSES DE DEPERISSEMENT

\begin{tabular}{clc}
\hline $\begin{array}{c}\text { Classe de } \\
\text { deperissement }\end{array}$ & \multicolumn{1}{c}{ Description sommaire } & $\begin{array}{c}\text { Equivalence } \\
\text { numerique }\end{array}$ \\
\hline 1 & Sujets sains. & 1 \\
2A & Petites feuilles, symptômes saisonniers. & 2 \\
2B & Symptomes permanents et plus apparents que 2B. & 3 \\
3A & Rameaux du sommet sans feuilles. & 4 \\
3B & Moins de 50\% de la cime affectée. & 5 \\
4A & Plus de 50\% de la cime morte. & 6 \\
4B & Seuls quelques rameaux vivants à la base de la cime. & 7 \\
5 et 6 & Sujets morts. & 8 \\
\hline
\end{tabular}


cependant, donné des équivalences numériques à ces classes de dépérissement. Les données sur les divers facteurs du microclimat proviennent de thermographes et d'hygrographes sous abri, d'évaporimètres Wright, de pluviomètres et de thermomètres dans le sol convenablement installès au centre de chaque aire. En outre, nous avons disposé, à deux endroits dans chaque aire, des couples de cellules Colman avec thermistor (1948) à 3,6 et 12 pouces de profondeur pour déterminer la température et le degré d'humidité du sol. A l'aide d'un ohmmètre portatif Berkeley, modèle 300, on obtient une valeur qui, une fois transformée en ohms et corrigée par un facteur de température, sert d'indice d'humidité. Pendant les quatre premières années, les données microclimatiques furent recueillies quotidiennement de la mi-mai à la miseptembre, et une fois la semaine ou à des intervalles plus courts au milieu de l'été de 1959.

\section{De cette série dobservations, il ressort,}

De cette série d'observations, il ressort, en premier lieu, que la saison estivale de 1955 a été notablement plus chaude que celles des trois années suivantes et que la température moyenne en juillet 1959 s'est maintenue à un niveau comparable à celui du même mois en 1955. A ce sujet, on peut noter que les moyennes mensuelles des maximums journaliers de la température de l'air ont dépassé $70^{\circ} \mathrm{F}$ pendant une grande partie de l'été en 1955 et au cours du mois de juillet en 1959, tandis que les moyennes des même périodes des annèes 1956, 1927 et 1958 se sont tenues à des paliers nettement moins élevés (Tableau 2). Les moyennes mensuelles des minimums journaliers soulignent aussi des différences marquées entre les étés 1955 et 1959, d'une part, et ceux des trois autres années, d'autre part. En effet, ces minimums moyens se situent le plus souvent près ou au-delà de $50^{\circ} \mathrm{F}$ en 1955 et 1959 et entre 39 et $48^{\circ} \mathrm{F}$ pendant les autres saisons d'été. Les moyennes journalières obtenues des thermogrammes montrent encore mieux les écarts entres les années, car les moyennes mensuelles ont varié entre 60 et $62^{\circ} \mathrm{F}$ en 1955 , entre 57 et $62^{\circ} \mathrm{F}$ en juillet et août 1959 , et entre 50 et $59^{\circ} \mathrm{F}$ en 1956,1957 et 1958 (Tableau 3). Notons aussi que ces moyennes ont été plus élevés dans l'aire $B$ que dans l'aire A.

Pour caractériser l'humidité de l'air, on peut se servir de la moyenne de l'humidité relative et du volume de la précipitation. Le Tableau 4 montre, notamment, que la moyenne hygrométrique de l'air dans les deux aires s'est maintenue à des niveaux nettement moins élevés en juillet 1955 et 1959 et en août 1959 que pendant les mêmes mois des autres années (Tableau 4). Ceci indique qu'en juillet 1955 et pendant une plus longue période en 1959, un climat plus sec que pendant les mêmes mois des autres années a régné dans cette station. Les mesures pluviométriques confirment, d'ailleurs, cette différence puisque la précipitation totale du 1er juin au 15 août n'a pas dépassé 5 pouces en 1955 et 1959, alors qu'elle s'est totalisée à plus de 7 pouces pendant la même période les autres années. Précisons cependant que les précipitations ont été notablement faibles du 16 juin au 13 juillet et du 19 juillet au 7 août en 1955 et du 6 au 27 juillet en 1959 (Tableau 5) comparées à celles des mêmes périodes des autres années.

Dans cette station, la température du sol a suivi d'assez près les grandes variations de la température de l'air. Toutefois l'amplitude des fluctuations a décru avec la profondeur. En 1955 et 1959, la température n'a jamais excédé 
$60^{\circ} \mathrm{F}$ à 12 pouces dans le sol, mais elle a atteint $63^{\circ} \mathrm{F}$ à 3 pouces pendant les périodes les plus chaudes (Tableau 6). En revanche, la température, même à 3 pouces de profondeur, n'a jamais dépassé $60^{\circ} \mathrm{F}$ pendant les trois autres années d'observation. Signalons en outre que le sol dans l'aire B, plus exposé aux ardeurs du soleil, s'est tenu légèrement plus chaud que dans l'aire $A$, mais cela seulement jusqu'à 3 pouces de profondeur.

\section{TABLEAU 2}

MOYENNES MENSUELLES DE LA TEMPERATURE (MAXIMUM ET

MINIMUM) DE L'AIR DURANT LES MOIS D'ETE DE 1955 A 1959, DANS DEUX aIRes D'Etudes A et B, a SaINT-Tite-Des-Caps

\begin{tabular}{|c|c|c|c|c|c|c|c|c|c|c|c|c|}
\hline \multirow[b]{4}{*}{ Annee } & \multicolumn{12}{|c|}{ Moyenne de la temperature en $\circ F$} \\
\hline & \multicolumn{6}{|c|}{ Maximum } & \multicolumn{6}{|c|}{ Minimum } \\
\hline & \multicolumn{2}{|c|}{ Juin } & \multicolumn{2}{|c|}{ Juillet } & \multicolumn{2}{|c|}{ Aout } & \multicolumn{2}{|c|}{ Juin } & \multicolumn{2}{|c|}{ Juillet } & \multicolumn{2}{|c|}{ Aout } \\
\hline & $\bar{A}$ & $\mathbf{B}$ & $A$ & B & A & B & $\mathbf{A}$ & B & $A$ & B & $\mathbf{A}$ & $\overline{\mathbf{B}}$ \\
\hline 1955 & 69 & 72 & 71 & 75 & 70 & 72 & 49 & 48 & 49 & 49 & 50 & 50 \\
\hline 1956 & 62 & 66 & 64 & 69 & 69 & 70 & 44 & 44 & 46 & 46 & 47 & 45 \\
\hline 1957 & 68 & 69 & 66 & 69 & 63 & 67 & 45 & 44 & 48 & 46 & 45 & 41 \\
\hline 1958 & 60 & 63 & 67 & 70 & - & - & 39 & 38 & 48 & 48 & - & - \\
\hline 1959 & 61 & 63 & 71 & 75 & 65 & 67 & 45 & 43 & 53 & 51 & 50 & 49 \\
\hline
\end{tabular}

TABLEAU 3

MOYENNES MENSUELLES DE LA TEMPERATURE DE L'AIR D'APRES LES MOYENNES JOURNALIERES (THERMOGRAMMES), AU COURS DES MOIS D'ETE DE 1955 A 1959, DANS DEUX AIRES D'etude A ET B, a SaINT-Tite-des-Caps

\begin{tabular}{|c|c|c|c|c|c|c|}
\hline \multirow[b]{3}{*}{ Annee } & \multicolumn{3}{|c|}{ Moyennes mensuelles d } & \multicolumn{3}{|c|}{ mperatur } \\
\hline & \multicolumn{3}{|c|}{ Aire $\mathbf{A}$} & \multicolumn{3}{|c|}{ Aire B } \\
\hline & Juin & Juillet & Aout & Juin & Juillet & Aont \\
\hline 1955 & 59.9 & 60.7 & 62.0 & 61.1 & 62.3 & 62.4 \\
\hline 1956 & 53.9 & 54.9 & 58.1 & 55.3 & 56.4 & 59.8 \\
\hline 1957 & 56.6 & 57.2 & 54.5 & 57.2 & 58.0 & 55.5 \\
\hline 1958 & 49.1 & 57.8 & - & 50.7 & 59.7 & - \\
\hline 1959 & 52.7 & 61.8 & 57.0 & 52.1 & 62.0 & 57.2 \\
\hline
\end{tabular}

TABLEAU 4

MOYENNES MENSUELLES DE L'HUMIDITE RELATIVE DE L'AIR D'APRES LES MOYENNES JOURNALIERES (HYGROGRAMMES), AU COURS DES MOIS D'ETE DE 1955 a 1959, Dans deux aires d’etUde A Et B, a Saint-Tite-Des-Caps

\begin{tabular}{|c|c|c|c|c|c|c|}
\hline \multirow[b]{3}{*}{ Annee } & \multirow{2}{*}{\multicolumn{3}{|c|}{$\begin{array}{l}\text { Moyennes mens } \\
\text { Aire } A\end{array}$}} & \multicolumn{3}{|c|}{ d'humidite relative } \\
\hline & & & & & Aire B & \\
\hline & Juin & Juillet & Aout & Juin & Juillet & Aout \\
\hline 1955 & 74.1 & 69.1 & 75.3 & 74.1 & 69.4 & 76.1 \\
\hline 1956 & 73.3 & 80.3 & 76.7 & 70.8 & 79.0 & 75.2 \\
\hline 1957 & 74.4 & 79.6 & 78.0 & 72.3 & 80.1 & 79.4 \\
\hline 1958 & 68.9 & 76.1 & - & 69.7 & 76.7 & - \\
\hline 1959 & 70.1 & 66.5 & 69.3 & 66.9 & 62.8 & 66.0 \\
\hline
\end{tabular}




\section{TABLEAU 5}

Pluviosite PENDANT DES PERTODES DE SECHERESSE EN 1955 ET 1959 COMPAREE AVEC CELLE DES PERIODES CORRESPONDANTES DES ANNEES 1956, 1957 et 1958 dans deuX aires d'ETUde a SAINT-Tite-des-CaPS

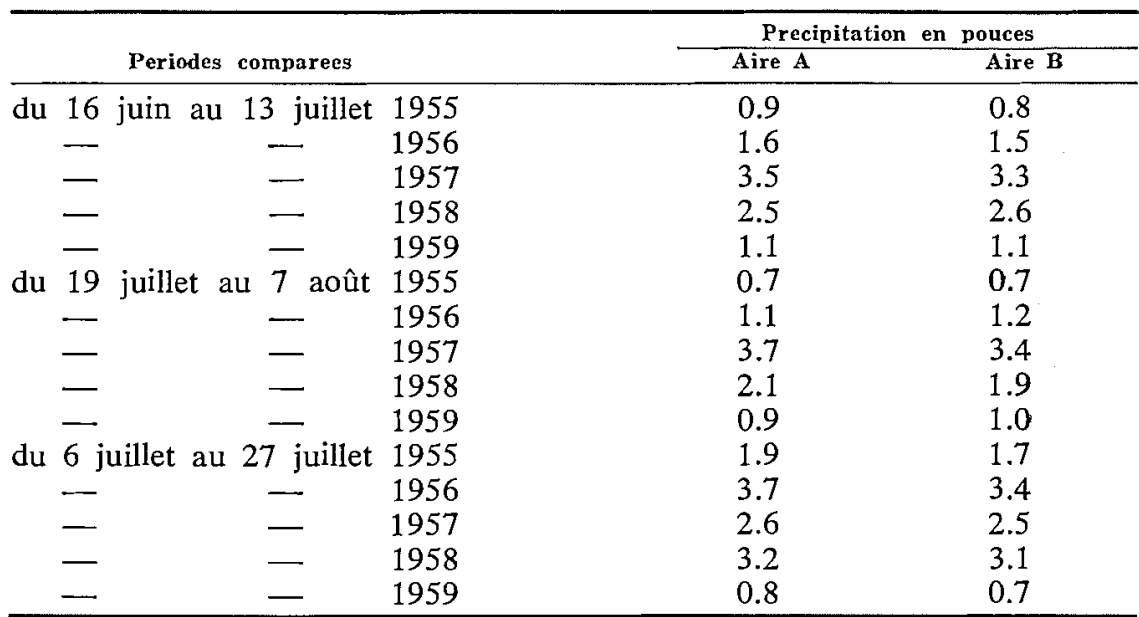

TABLEAU 6

Periodes de temperature superieure a $60^{\circ} \mathrm{F}$ a difFerentes profondeurs DANS LE SOL, DANS AIRES D'ETUde, A SAINT-TrTe-DEs-Caps, DE 1955 a 1959 INCLUSIVEMENT

\begin{tabular}{cccccc}
\hline Annee & $\begin{array}{c}\text { Aire } \\
\text { d'etude }\end{array}$ & $\begin{array}{c}\text { Profondeur } \\
\text { en pouces }\end{array}$ & \multicolumn{1}{c}{ Periode } & $\begin{array}{c}\text { Temperature } \\
\text { maximum en }\end{array}{ }^{\circ} \mathbf{F}$ \\
\hline 1955 & A & 3 & du 20 juillet au 25 juillet & 62 \\
& & 6 & du 21 juillet au 22 juillet & 60.5 \\
& & 3 & du 13 août au 24 août & 63 \\
& & 6 & du 17 août au 24 août & 62 \\
& B & 3 & du 6 juillet au 11 juillet & 61.5 \\
& & 3 & du 16 juillet au 27 juillet & 63 \\
& & 3 & du 2 août au 7 août & 62 \\
1959 & A \& B & 3 & du 12 août au 24 août & 63 \\
& 3 & du 27 juillet au 1 août & 63 \\
\hline
\end{tabular}

En ce qui concerne l'humidité du sol, nous avons adopté la résistance électrique comme mesure représentative de ce facteur à l'instar de Fraser (1957a), après avoir calibré les cellules de Colman (1958) avec du sol prélevé aux endroits mêmes où elles étaient placées. Ainsi, nous avons déterminé que 4,000 ohms représentaient le point de saturation et 100,000 ohms approximativement le point de flétrissement du sol dans ces aires d'étude.

Les données recueillies au cours de ces cinq années montrent que le degré d'humidité du sol a varié selon l'intensité et la fréquence des précipitations. En général, la résistance électrique s'est maintenue aux environs de 5,000 ohms 
et ne s'est élevée que pendant de courtes périodes jusqu'à 7,000 ohms en 1956, 1957 et 1958; ce qui indique bien que le sol dans les deux aires a conservé un haut degré d'humidité au cours de ces trois années. Mais, aux périodes de faible précipitation et de température élevée ont correspondu des jours de sécheresse du sol. Ainsi, la faible précipitation de 0.9 pouce et la température élevée du 16 juin au 13 juillet 1955 se sont traduites par la desiccation rapide du sol qui a dépassé le point de flétrissement $(100,000 \mathrm{ohms})$ pendant une journée à 3 pouces de profondeur dans l'aire $B$ et qui a atteint $60,000 \mathrm{ohms}$ dans l'aire A (Fig. 1). Lors d'une autre période sèche, du 19 juillet au 7 août 1955, les pluviomètres n'ont reçu que 0.7 pouce d'eau et la résistance a atteint 50,000 ohms dans l'aire A et environ 8,500 dans l'aire B. En 1959, trois semaines de chaleur et de faible pluviosité ont aussi causé un assèchement du sol dont le point culminant de la résistance n'a pas dépassé la cote de 40,000 ohms dans l'aire A et de 30,000 ohms dans l'aire B. En outre, notons qu'à 6 et 12 pouces de profondeur, la résistance des cellules Colman n'a monté au-delà de 20,000 ohms que pendant deux laps de temps en 1955 et aussi que la desiccation en profondeur a été plus forte dans l'aire A sous un couvert forestier plus dense que dans l'aire B plus exposée.

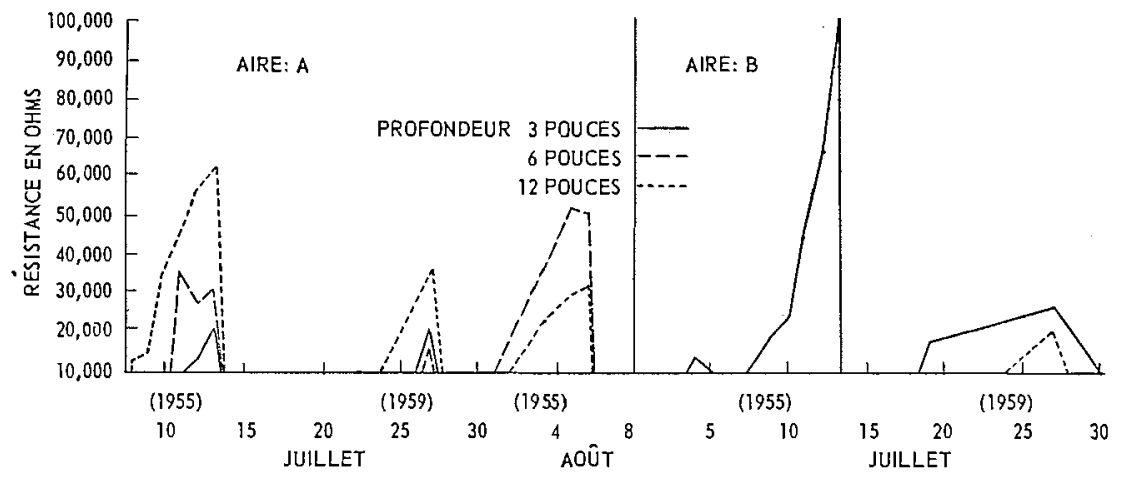

FIGURE 1. Mesures de résistance des cellules Colman supérieures à 10,000 ohms à Saint-Titedes-Caps, pendant les périodes sèches de 1955 et de 1959.

\section{La Maladie}

Lorsque ces observations débutèrent en 1955 les bouleaux présentaient depuis plusieurs années des symptômes plus ou moins prononcés de dépérissement. Cependant, les arbres de l'aire B avaient souffert beaucoup plus de ce mal que ceux de l'aire $A$, comme l'indique l'équivalence numérique moyenne de 6.5 (4A et $4 \mathrm{~B}$ ) pour la première et de $5(3 \mathrm{~B})$ pour la deuxième (Tableau 7). Au cours des cinq années subséquentes, le dépérissement moyen a marqué un léger progrès jusqu'en 1958 et il est resté stationnaire par la suite, comme on peut le constater dans le même tableau. Outre la progression presqu'insensible des symptômes chez l'ensemble des arbres dans chacune des aires au cours de la période d'observation, les mutations de classe de dépérissement entre 1955 et 1960 peuvent aussi fournir des renseignements sur l'évolution de la maladie. Par l'analyse des données prélevées dans les 
deux aires, on constate, en premier lieu (Tableau 8), que le pourcentage des mutations a augmenté progressivement surtout chez les arbres à dépérissement avancé en 1955. Ainsi, les sujets assez fortement atteints (3A et plus) en 1955 ont continué à dépérir dans une proportion de 54 à 68 pour cent, tandis que les arbres placés dans les classes 1, 2A et 2B en 1955 n'ont passé par la suite dans des catégories plus avancées que dans la proportion de 16,25 et 31 pour cent respectivement. Mentionnons aussi que 8 arbres des classes 2A, 3A, 3B et 4B ont montré des signes de recouvrement de santé. Enfin, ces résultats révèlent que 3.5 pour cent des arbres des classes supérieures ( 1 à 2B), 13.2 pour cent de ceux des classes moyennes ( $3 \mathrm{~A}$ et $3 \mathrm{~B}$ ) et 38.3 pour cent des classes inférieures (4A et 4B) sont entrés dans les catégories 5 et 6 réservées aux sujets morts. En somme, en six ans, le progrès du dépérissement a

\section{TABLEAU 7}

EQUIVALENCE NUMERIQUE MOYENNE DU DEPERISSEMENT DU BOULEAU A PAPIER DANS DEUX D'ETUde, A SAINT-TITE-DES-CAPS, DE 1955 A 1960

\begin{tabular}{cccccccc}
\hline \multirow{2}{*}{$\begin{array}{c}\text { Aire } \\
\text { d'etade }\end{array}$} & $\begin{array}{c}\text { Nombre total } \\
\text { de bouleaux }\end{array}$ & \multicolumn{5}{c}{ Equivalence } & numerique \\
\cline { 3 - 8 } & 258 & 5.0 & 5.2 & 5.4 & 5.5 & 5.6 & 5.6 \\
A & 301 & 6.5 & 6.6 & 6.7 & 6.8 & 6.8 & 6.8 \\
\hline
\end{tabular}

\section{TABLEAU 8}

MUTATION DE CLASSES DE DEPERISSEMENT DE 559 BOULEAUX A PAPIER PENDANT LA PERIODE 1955-1960, DANS DEUX AIRES D'ETUDE, A SAINTE-TITE-DES-CAPS

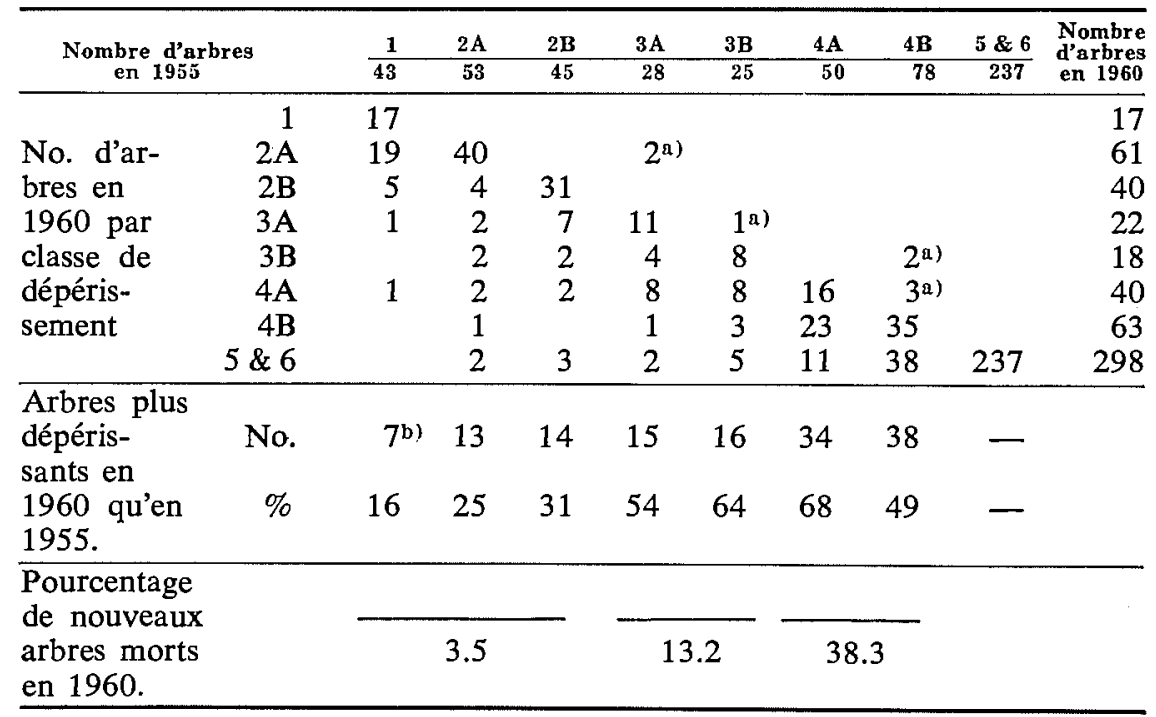

a) Arbres dont l'état de santé s'est améliorć entre 1955 et 1960.

b) Les mutations de 1 à $2 \mathrm{~A}$ ne sont pas considérées irréversibles. 
été plutôt faible chez les sujets relativement sains en 1955 et un peu plus élevé chez les arbres dont la santé était assez fortement ébranlée au début des observations.

\section{CONCLUSIONS}

Cinq années d'observations sur le microclimat estival dans un peuplement de bouleau à papier ont fourni les principaux résultats suivants: (1) $\mathrm{Si}$ la température maximum de l'air a touché $95^{\circ} \mathrm{F}$, les moyennes journalières n'ont pas dépassé $70^{\circ} \mathrm{F}$ pendant les périodes les plus chaudes; (2) La température du sol, qui, le plus souvent, n'a pas dépassé $60^{\circ} \mathrm{F}$ à 3 pouces de profondeur, a monté à peine jusqu'à $63^{\circ} \mathrm{F}$ pendant quelques jours en 1955 et en 1959; (3) Hormis certaines périodes en 1955 et en 1959, les saisons d'été ont été plutôt humides pendant ces cinq années; (4) En général, le sol s'est maintenu à un niveau d'humidité très favorable à la végétation, sauf pendant deux périodes sèches en 1955 et une en 1959 où la sécheresse a atteint un palier critique pendant quelques jours. Ces constatations corroborent celles que Fraser (1957b) a dérivées d'une étude analogue dans un peuplement de bouleau jaune en Ontario. A cet endroit, en août 1953, la température du sol a touché $65^{\circ} \mathrm{F}$ à 2 pouces et $62^{\circ} \mathrm{F}$ à 3 pouces dans un sol dont le régime d'humidité était semblable ( 2 d'après la classification de Hills) à celui où nous avons poursuivi nos observations. Cet auteur (Fraser 1957a) a également relevé des conditions d'humidité du sol comparables à celles que nous avons observées dans le Québec et noté, comme nous, des périodes de sécheresse assez avancée quand la pluviosité est restée faible. Si l'on admet, avec Fraser (1957a), qu'au-delà de $50,000 \mathrm{ohms}$ la résistance dénote une situation extrême du point de vue de la végétation, des sécheresses véritables ont eu lieu à Saint-Tite-des-Caps en 1955.

Pendant la même période d'observation à cet endroit, le climat estival ne semble pas avoir modifié d'une manière appréciable la santé des bouleaux. En effet, les symptômes ne se sont guère intensifiés au cours de ces six années et le déclin graduel d'un certain nombre de sujets s'est manifesté surtout chez les individus les plus faibles (Lortie, 1960) et déjà mal en point au début des observations. De plus, les saisons chaudes et sèches de 1955 et 1959 n'ont pas laissé de traces de leurs effets nocifs sur le feuillage. Enfin, pendant ce laps de temps, aucune différence notable de comportement n'a été observée entre les arbres des deux aires bien que les conditions de température et d'humidité du sol aient été quelque peu plus critiques dans un cas. Il convient aussi de mentionner ici que ces résultats obtenus dans un peuplement de bouleau à papier dans l'est du Québec concordent assez bien avec ceux consignés par Fraser (1959) en Ontario, mais dans un peuplement de bouleau jaune relativement en bon état de santé au départ.

Il ressort de l'ensemble de ces observations que les variations assez prononcées du microclimat pendant les mois d'été ne paraissent pas avoir affecté les bouleaux dans un endroit où le dépérissement a régné d'une manière plutôt intense plusieurs années auparavant. Cette notion s'était du reste accréditée par les travaux de Fraser (1957a, b) en Ontario et aussi par ceux Clark et Barter (1958) au Nouveau-Brunswick. Ces derniers, par des études statistiques du climat dans les Provinces Maritimes, n'ont pu trouver d'indications 
que les périodes chaudes et sèches pendant les mois d'été avaient exercé une influence néfaste chez les bouleaux.

\section{REMERCIEMENTS}

Nous sommes reconnaissants envers Messieurs Lionel Daviault et PaulEmile Vézina d'avoir revu le manuscrit.

CLARK, J. et G. W. BARTER. 1958. Growth and climate in relation to dieback in yellow birch. Forest Sci. 4: 343-364.

COLMAN, E. A. 1948. Manual of instructions for use of the fiberglass soil moisture instrument. Berkeley Scientific Co., Richmond, California, 19 pp.

FRASER, D. A. 1957a. Annual and seasonal march of soil moisture under a hardwood stand. Forest Res. Div., Forestry Branch, Canada Dept. Northern Affairs and Nat. Resources, Tech. Note 55.

FRASER, D. A. 1957b. Annual and seasonal march of soil temperature on several sites under a hardwood stand. Forest Res. Div., Forestry Branch, Canada Dept. Northern Affairs and Nat. Resources, Tech. Note 56.

FRASER, D. A. 1959. Nine years of observations on the condition of 241 yellow birch. Forest Res. Div., Forestry Branch, Canada Dept. Northern Affairs and Nat. Resources, Tech. Note 69.

HAWBOLDT, L. S. 1952. Climate and birch "dieback". Nova Scotia Lands and Forests, Bull. 6.

HAWBOLDT, L. S. et A. SKOLKO. 1948. Investigations of yellow birch dieback in Nova Scotia in 1947. J. Forestry 46: 659-671.

HILLS, G. A. 1945. A decimal system for the classification and mapping of Ontario soils. Sci. Agric. 25: 253-272.

LINTEAU, A. 1959. Classification des stations forestières de la section des conifères du nord-est, région forestière boréale du Québec. Direct. des forêts, Min. du Nord Canadien et des Res. Nat., Bull. 118.

LORTIE, M. 1960. Rapport entre le dépérissement des cimiers et le diamètre des arbes dans des peuplements de bouleaux à papier. Bull. d'inf. bimestr. Serv. Recherches, Div. Biol. forestière, Min. Agr. du Canada, 16 (6): 1.

POMERLEAU, R. 1944. Observations sur quelques maladies non parasitaires des arbres dans le Québec. Can. J. Res. C, 22: 171-189.

REDMOND, D. R. 1955. Studies in forest pathology. XV. Rootlets, mycorrhiza, and soil temperatures in relation to birch dieback. Can. J. Botany 33: 595-627.

REDMOND, D. R. 1957. The future of birch from the viewpoint of disease and insects. Forestry Chron. 33: 25-30.

REDMOND, D. R. 1958. Températures du sol et dépérissement du bouleau. Bull. d'inf. bimestr. Serv. Sciences, Div, Biol. forestière, Min. Agr. du Canada, 14 (5): 1. 